Med Klin Intensivmed Notfmed 2014 .

109:154-155

DOI 10.1007/s00063-013-0301-8

Online publiziert: 22. März 2014

๑) Springer-Verlag Berlin Heidelberg 2014

\section{Engelmann ${ }^{1} \cdot$ R. Bellmann ${ }^{2}$}

${ }^{1}$ Leipzig

${ }^{2}$ Arbeitsgruppe Klinische Pharmakokinetik, Labor für Inflammationsforschung, Gemeinsame Einrichtung Internistische Notfall- und Intensivmedizin, Department Innere Medizin, Medizinische Universität Innsbruck

\title{
Adäquate Antibiotikatherapie auf der Intensivstation
}

Infektionen und Sepsis beherrschen den intensivmedizinischen Alltag und machen Antibiotika zur wichtigsten Medikamentengruppe auf Intensivstationen. Die Herausgeber der Zeitschrift, die im Jahr 2012 das Themenheft Grundwissen zur Antibiotikatherapie planten, hatten in ihrer täglichen klinischen Praxis übereinstimmend Defizite im Wissen über und zum Umgang mit Antibiotika ausgemacht. Diese schlagen sich besonders in einer inadäquaten Initialtherapie nieder und beeinflussen darüber hinaus den gesamten Behandlungszeitraum.

\section{) Inadäquate Initialtherapie trägt zu erheblicher Übersterblichkeit bei}

Inadäquate Initialtherapie trägt $\mathrm{zu}$ erheblicher Übersterblichkeit bei. Die Rate inadäquater Behandlungen im stationären Bereich beträgt derzeit 20-70 \%. Inadäquate Antibiotikatherapie wurde zudem als eine wichtige Ursache von $\mathrm{Re}$ sistenzentwicklung und nosokomialer Infektion ausgemacht. Die Prävalenz von Bakterien, die Extended-spectrum- $\beta$-Lactamasen (ESBL) bilden, hat sich zwischen 2002 und 2009 verfünffacht. Mindestens 25.000 Menschen sterben jährlich im Gebiet der Europäischen Union an Infektionen mit multiresistenten Erregern, 3- und 4-fach multiresistente gramnegative Erreger (MRGN) sind Wortschöpfungen der letzten Jahre. Die Senkung der Sterblichkeit an schwerer Sepsis und septischem
Schock stagniert bei etwa $50 \%$. Licht im Tunnel ist nicht zu erkennen, erst recht nicht, wenn in den Jahren 2008-2012 nur 2 Antibiotikaneuzulassungen erfolgten.

Vor diesem dramatischen Hintergrund erfolgt tägliches ärztliches Handeln. Die Philosophie im Umgang mit Antibiotika muss sich ändern und dieses Themenheft soll dazu Grundwissen vermitteln.

Im Zentrum des Themenhefts steht der Beitrag zur „Tarragona-Strategie“. Er transformiert die für die Therapie der nosokomialen Pneumonie geschaffene Strategie auf die generelle Antibiotikatherapie in der Intensivstation. Durch strukturiertes Denken entlang eingängiger Schlagworte wird die Mobilisation ärztlichen Wissens in Akutsituationen erleichtert und sog. chaotisches Denken unterdrückt. Wesentliche Aspekte dieses klinischen Beitrags werden im Themenheft spezieller beleuchtet, um das Verständnis für die Forderungen der sog. TarragonaStrategie zu vertiefen.

Das betrifft zunächst die Pharmakokinetik und Pharmakodynamik sowie deren Spezifik bei kritisch Kranken. Es erfolgen diesbezügliche Zielvorgaben und Dosierungsempfehlungen beim Einsatz kontinuierlicher Nierenersatzverfahren auf der Intensivstation.

Im Weiteren werden Wirkprinzipien der Antibiotika, Resistenzmechanismen und Indikationsgebiete einzelner Antibiotikagruppen dargestellt. Der Beitrag widmet sich auch dem Sinn von Kombinationstherapien, die bei zunehmender
Resistenzentwicklung wieder mehr in den Fokus des Klinikers rücken.

\section{) Kombinationstherapien rücken bei zunehmender Resis- tenzentwicklung in den Fokus}

Für den Erfolg einer antibiotischen Therapie ist entscheidend, ob die notwendige Antibiotikakonzentration am Ort der Infektion erreicht wird. Bei kritisch Kranken besteht eine erhöhte Variabilität der Gewebepenetration gegenüber Gesunden. Das Wissen darüber ist lückenhaft. Es werden klinisch bereits einsetzbare Verfahren dargestellt, Gewebekonzentrationen der Antibiotika am Ort der Infektion zu messen.

Schließlich unterzieht der Mikrobiologe die Verfahren der Resistenzbestimmung einer kritischen Betrachtung. Das Fazit geht dahin, dass der Kliniker nicht nur das kategorisierte Testergebnis als sensibel, intermediär oder resistent, sondern auch die minimale Hemmkonzentration (MHK) vor dem Hintergrund des Testverfahrens seines örtlichen Labors kennen und interpretieren können muss. Die Kategorisierung sollte nach Empfehlung von European Committee on Antimicrobial Susceptibility Testing (EUCAST) erfolgen.

Schließlich werden klinische Befunde und Laborparameter vermittelt, nach denen eine antibiotische Therapie deeskaliert oder beendet werden kann. Eine üblicherweise zu langdauernde antibioti- 
sche Therapie birgt die Gefahr von Selektion und Resistenzentwicklung mit allen Folgen, über die das Themenheft Wissen vermitteln will. Antibiotikakosten spielen eine sekundäre Rolle. Die Therapie wird dann kostspielig, wenn keine adäquate Antibiotikatherapie erfolgt.

\section{Korrespondenzadressen}

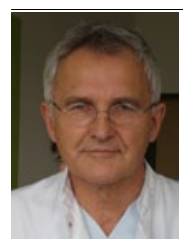

Prof. em. Dr. L. Engelmann

Sigebandweg 25,

04279 Leipzig

pdle@gmx.de

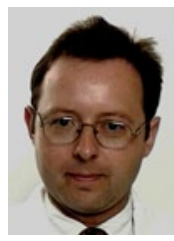

Ao. Univ. Prof. Dr. R. Bellmann Arbeitsgruppe Klinische Pharmakokinetik, Labor für Inflammationsforschung, Gemeinsame Einrichtung Internistische Notfall- und Intensivmedizin, Department Innere Medizin, Medizinische Universität Innsbruck

Anichstraße 35,

A-6020 Innsbruck

Österreich

romuald.bellmann@

i-med.ac.at

\section{Einhaltung ethischer Richtlinien}

Interessenkonflikt. L. Engelmann gibt an, Teilnehmer eines infektiologischen Expertenworkshops der Fa. Novartis zu sein. R. Bellmann gibt an, Forschungsunterstützungen und Vortragshonorare von den Firmen Chiesi Pharmaceuticals, Pfizer und Merck, Sharp \& Dohme erhalten zu haben.

\section{Netter, Frank H.}

\section{Netters Innere Medizin}

Stuttgart: Georg Thieme Verlag 2013, 848 S., 1757 Abb., (ISBN 978-3-13-123962-4), gebunden, 99.99 EUR

Frank Netter ist der bekannteste medizinische Illustrator. Generationen von Medizinstudenten haben in Vorlesungen seine einprägsamen klinischen und pathophysiologischen Abbildungen gesehen, und er hat maßgeblich dazu beigetragen, dass Ärzte Diagnosen sicher stellen können und komplexe Zusammenhänge rasch verstehen.

In der nun vorliegenden 2. Auflage von Netters Innere Medizin wurden die wichtigsten seiner Zeichnungen in einem Band zusammengefasst und durch moderne klinische und diagnostische Abbildungen ergänzt. Der Begriff Innere Medizin ist hier weitgefasst, denn auch wichtige neurologische und pädiatrische Erkrankungen werden dargestellt. Auf der jeweils rechten Buchseite findet man die Abbildungen und auf der dazugehörigen linken Buchseite kurze Texte zu Anatomie, Pathophysiologie, Klinik, Diagnostik und Therapie. Die Texte wurden von erfahrenen Klinikern verfasst. Das Buch eignet sich sehr gut für Prüfungen jeder Art, vom Staatsexamen bis hin zur Facharztprüfung. Durch die Prägnanz der Abbildungen ist das Buch auch für Naturwissenschaftler geeignet, die einen Einblick in die Klinik gewinnen möchten, und auch interessierte Laien, die sich gezielt über ein Thema informieren möchten, werden das Buch mit Gewinn lesen.

Das Buch stellt eine ideale Ergänzung zu klassischen Lehrbüchern der Inneren Medizin dar. Es besteht keine Konkurrenzsituation, denn der Netter lebt von der Lebendigkeit der Abbildungen. Allerdings sind manche Zeichnungen aus den Atlanten sehr stark verkleinert und die Details nur schwer erkennbar. Und der Zeitraum zwischen der Entstehung der Abbildungen und der Gegenwart wird immer größer, so dass manche Abbildungen etwas antiquiert aussehen. Aber dennoch gehe ich davon aus, dass es noch weitere Auflagen des Netter geben wird. Abgerundet wird das auf sehr gutem Papier gedruckte und stabil gebundene Buch durch ein umfassendes aktuelles Verzeichnis mit weiterführender Literatur und ein kompaktes Sachverzeichnis. Das Preis-Leistungsverhältnis ist mit knapp 100 Euro angemessen. Das Gewicht des Bu- ches mit über $2 \mathrm{~kg}$ macht es aber nur bedingt transportfähig. Für die nächste Auflage wäre eine e-book-Version sehr wünschenswert. Man könnte sich dann gezielt kleine Abbildungen vergrößern.

R. Seifert (Hannover) 